\title{
A novel approach to the teaching of planar mechanism dynamics - a case study
}

\author{
Rosario Sinatra ${ }^{a}$ and Jorge Angeles ${ }^{b}$ \\ ${ }^{a}$ Dipartimento di Ingegneria Industriale e Meccanica, Facoltà d'Ingegneria, \\ Università di Catania, Viale A. Doria 6, 95125 Catania, Italy \\ ${ }^{\mathrm{b}}$ Department of Mechanical Engineering \& Centre for Intelligent Machines, \\ McGill University, Montreal, Quebec, Canada H3A 2K6 \\ E-mail: rsinatra@diim.uniet.it
}

\begin{abstract}
We propose a novel approach to the teaching of undergraduate planar mechanism dynamics. To illustrate the approach, we use a case study, the dynamics of the planar slider-crank mechanism. In this case study, we make extensive use of an operator representing in two-dimensional form the cross-product of two vectors. Furthermore, by using the natural orthogonal complement, introduced elsewhere, we produce a systematic procedure to derive a dynamic model of the same class of mechanism. Subsequently, we illustrate how, with the use of the aforementioned operator, the dynamic balancing of this mechanism, as first proposed by Berkof and Lowen for RRRR planar linkages, and extended by Bagci to the slider-crank mechanism, simplifies tremendously.
\end{abstract}

Keywords dynamic balancing; planar slider-crank mechanism; undergraduate teaching

The teaching of the planar kinematics and dynamics of machines has undergone very few, if any, innovations in the last half century of what has been called modern mechanism and machine theory [1]. This epoch has been marked by the advent of the computer, in which has been traditionally called theory of machines and mechanisms (TMM), and nowadays is being called mechanism and machine science (MMS). If we compare MMS textbooks of the late 1940s or early 1950s with current ones, a remarkable difference is that the latter include, as a rule, code (usually Fortran or BASIC) to evaluate, verbatim, classical formulas. Needless to say, the casting of these formulas in computer code does not advance the state of the art in the teaching of MMS. A recent book [2] does intensively use Matlab for the solution of MMS problems.

We have reviewed the formulation of planar kinematics and dynamics, and came across an alternative, novel formulation, based on an operator used to represent the three-dimensional cross-product in two-dimensional form. What the operator offers is an alternative to the popular treatment of planar kinematics and dynamics based on complex numbers. However, notice that complex numbers cannot handle threedimensional mechanism analysis; the method introduced here cannot only be readily extended to three dimensions, but was also derived from three-dimensional analyses. With the aid of the operator introduced here, the kinematic analysis of planar mechanisms is greatly simplified. The dynamic analysis of mechanisms is simplified likewise with the aid of this operator and the introduction of the natural orthogonal complement $[3,4]$.

Subsequently, we show how the equations for the dynamic balancing of the planar 


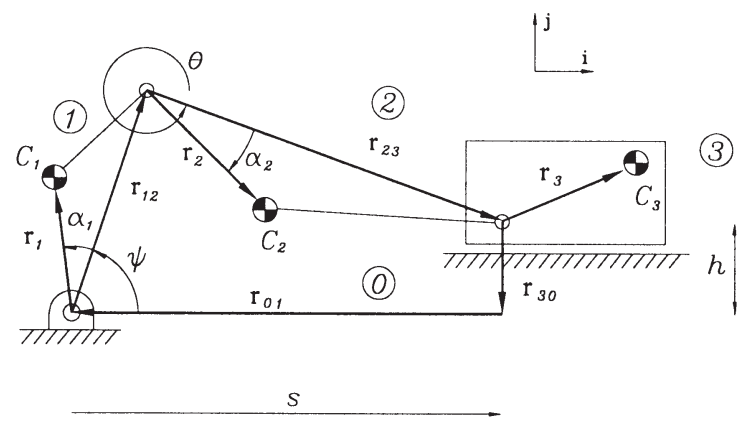

Fig. 1. Slider-crank mechanism.

slider-crank mechanism can be derived much more concisely with the use of the aforementioned operator, following a novel, simplified approach.

The dynamic balancing of linkages is a classical problem in MMS, which acquires special significance in high-speed machinery. Berkof and Lowen [5] proposed a general method of planar mechanism balancing. The method is general in the sense that it is applicable to any force-balanceable planar linkage, and provides the necessary and sufficient set of balancing equations. These authors used the principle that if the total centre of mass of a mechanism can be made stationary, then the shaking force vanishes. The approach followed by Berkof and Lowen, called the 'method of linearly independent vectors', allows the analyst to render the mass centre of the overall mechanism stationary.

As opposed to the complex vector method of Berkof and Lowen, a new method for complete balancing of planar linkages was proposed by Kochev [6], which applies ordinary vector algebra and produces the balancing equations in Cartesian form.

Numerous other researchers have investigated the problem of dynamic balancing [7-10]. More recently, research attention has been directed to the balancing of multi-degree-of-freedom machinery, in particular for planar linkages [11-12], while the balancing of spatial mechanisms has been studied as well $[14,15]$. The static balancing of serial manipulators using counterweights and springs has been studied [15-17], along with that of parallel manipulators [18-20].

The complete force and moment balancing of the planar slider-crank mechanism was reported by Bagci [21]. When in motion, the slider-crank mechanism, shown in Fig. 1, exerts forces and moments on the frame on which it is mounted, by virtue of the inertia of its links. These forces can be reduced by a proper mass redistribution of the moving links. Bagci used the method of linearly independent vectors of Berkof and Lowen's to balance the shaking force by the redistribution of the link mass. Bagci went on to balance the shaking moment by both the redistribution of the masses and by the addition of counter-rotary masses. Moreover, Nahon et al. [10] showed that the shaking moment cannot be balanced by means of a mass redistribution of the links alone, but by means of control. These authors proposed the use of redundant actuation in a dynamically balanced four-bar planar linkage to elimi- 
nate the shaking moment. Thümme [9] demonstrated experimentally the feasibility of shaking-force elimination. This paper deals with the dynamic balancing of the slider-crank mechanism using a new teaching aid. First, the mathematical modelling of the mechanism, using the natural orthogonal complement [3], is obtained. Then, two design equations in six design parameters for the complete dynamic balancing are derived.

\section{Mathematical modelling of the slider-crank mechanism}

The discussion below draws from basic concepts of linear algebra, which is nowadays a key component of the core undergraduate curriculum of mechanical engineering programmes in many universities. In our experience, undergraduate students tend to forget their linear algebra immediately after they have been exposed to it because, admittedly, they do not use it in their engineering science courses. A 60minute review of basic definitions and concepts usually suffices to bring them back. We believe that avoiding the use of linear algebra in the undergraduate engineering courses just contributes to the students' estrangement from the subject.

The kinematic constraints and the mathematical modelling of the slider-crank mechanism are obtained using the method of the natural orthogonal complement [3]. As applied to planar mechanisms, the method requires the definition of the twist, $\mathbf{t}_{i}$ of the $i$ th link and of the wrench, $\mathbf{w}_{i}$, exerted onto the same link as threedimensional arrays, namely,

$$
\mathbf{t}_{i} \equiv\left[\begin{array}{c}
\omega_{i} \\
\dot{\mathbf{c}}_{i}
\end{array}\right], \quad \mathbf{w}_{i} \equiv\left[\begin{array}{c}
T_{i} \\
\mathbf{f}_{i}
\end{array}\right]
$$

where $\omega_{i}$ and $T_{i}$ are the scalar angular velocity and the torque acting on the $i$ th link, respectively. Moreover, $\dot{\mathbf{c}}_{i}$ and $\mathbf{f}_{i}$ are the two-dimensional vectors representing the velocity of the mass centre of the $i$ th link and the force acting at that centre. Likewise, the $3 \times 3$ inertia dyad, represented by matrix $\mathbf{M}_{i}$, is defined as

$$
\mathbf{M}_{i} \equiv\left[\begin{array}{cc}
I_{i} & \mathbf{0}^{T} \\
\mathbf{0} & m_{i} \mathbf{1}
\end{array}\right]
$$

where $I_{i}$ is the polar moment of inertia of the $i$ th link about its mass centre. Moreover, $\mathbf{0}$ and $\mathbf{1}$ are the two-dimensional zero vector and the $2 \times 2$ identity matrix, respectively.

Below we represent the scalar moment $T$ exerted by a force $\mathbf{f}$ acting at a point $P$ of a rigid body, of position vector $\mathbf{p}$, with respect to a point $Q$, of position vector $\mathbf{q}$, in the form

$$
T=\mathbf{f}^{T} \mathbf{E}(\mathbf{p}-\mathbf{q})=-(\mathbf{p}-\mathbf{q})^{T} \mathbf{E f}
$$

where the $2 \times 2$ orthogonal matrix $\mathbf{E}$ represents a rotation in the plane through an angle of $90^{\circ}$ counter-clockwise, namely, 


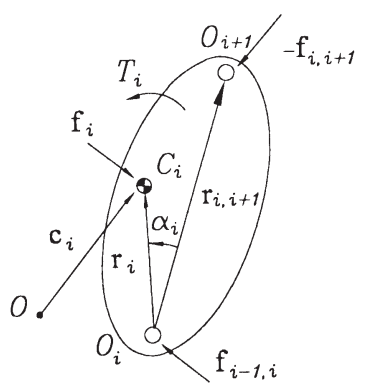

Fig. 2. Free-body diagram of the ith link.

$$
\mathbf{E} \equiv\left[\begin{array}{cc}
0 & -1 \\
1 & 0
\end{array}\right]
$$

Henceforth, $\mathbf{f}_{i-1, i}$ denotes the force exerted by the $(i-1)$ st link onto the $i$ th link, with $i-1$ understood as modulo 3 . Likewise, $T_{i-1, i}$ is defined as the couple exerted by the $(i-1)$ st link onto the $i$ th link.

Now, the Newton-Euler (N-E) equations for the $i$ th link moving in the plane, as depicted in Fig. 2, are written, for $i=1,2$, in the form

$$
\begin{aligned}
& \mathbf{r}_{i}^{T} \mathbf{E} \mathbf{f}_{i-1, i}+\left(\mathbf{r}_{i, i+1}-\mathbf{r}_{i}\right)^{T}-\mathbf{E} \mathbf{f}_{i, i+1}+T_{i}=I_{i} \dot{\omega}_{i} \\
& \mathbf{f}_{i-1, i}-\mathbf{f}_{i, i+1}+\mathbf{f}_{i}=m_{i} \ddot{\mathbf{c}}_{i}
\end{aligned}
$$

Upon combining equations (5) and (6), we obtain

$$
\mathbf{M}_{i} \dot{\mathbf{t}}_{i}=\mathbf{w}_{i}+\mathbf{B}_{i} \phi_{i}, \quad i=1,2
$$

where the $3 \times 4$ matrix $\mathbf{B}_{i}$ and the four-dimensional vector $\phi_{i}$ are defined as

$$
\mathbf{B}_{i} \equiv\left[\begin{array}{cc}
\mathbf{r}_{2}^{T} \mathbf{E} & \mathbf{b}_{i}^{T} \mathbf{E} \\
\mathbf{1} & -\mathbf{1}
\end{array}\right], \quad \phi_{i} \equiv\left[\begin{array}{c}
\mathbf{f}_{i-1, i} \\
\mathbf{f}_{i, i+1}
\end{array}\right]
$$

for $i=1,2$, with $\mathbf{b}_{i} \equiv \mathbf{r}_{i, i+1}-\mathbf{r}_{i}$. Note that $\phi_{i}$ denotes the array of internal or constraint forces acting on the $i$ th link. These are forces exerted by the neighbouring links; not by the environment. We will thus speak of internal or constraint wrenches to indicate the concurrent action of force and couple.

For link 3, note that, in order to account for the offset of the point of application of the reaction force, it is assumed that link 0 exerts on link 3 a force $-\mathbf{f}_{30}$ acting at $C_{3}$ and a couple $-T_{30}$. Moreover, in order to keep the discussion at an introductory level, we assume that all joints, revolute and prismatic, are ideal, i.e., they provide neither backlash nor friction. In this light, then, $-\mathbf{f}_{30}$ is normal to the surface of 


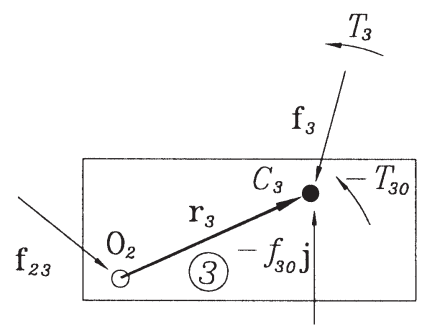

Fig. 3. Free-body diagram of the slider.

sliding. Figure 3 shows the free-body diagram of the slider, where we have $\mathbf{f}_{30}=f_{30} \mathbf{j}$. Thus, the N-E equations of link 3 are

$$
\begin{aligned}
& \mathbf{r}_{3}{ }^{T} \mathbf{E} \mathbf{f}_{23}-T_{30}+T_{3}=0 \\
& \mathbf{f}_{23}-f_{30} \mathbf{j}+\mathbf{f}_{3}=m_{3} \ddot{\mathbf{c}}_{3}
\end{aligned}
$$

which can be cast in the form

$$
\mathbf{M}_{3} \dot{\mathbf{t}}_{3}=\mathbf{B}_{3} \phi_{3}+\mathbf{w}_{3}
$$

where $\mathbf{M}_{3}$ and $\mathbf{B}_{3}$ are $3 \times 3$ and $3 \times 4$ matrices. Moreover, $\phi_{3}$ is a four-dimensional vector, while $\mathbf{t}_{3}$ and $\mathbf{w}_{3}$ are both three-dimensional vectors, all these items being displayed below:

$$
\mathbf{M}_{3} \equiv\left[\begin{array}{cc}
I_{3} & \mathbf{0}^{T} \\
\mathbf{0} & m_{3} \mathbf{1}
\end{array}\right], \quad B_{3} \equiv\left[\begin{array}{ccc}
\mathbf{r}_{3}^{T} \mathbf{E} & -1 & 0 \\
\mathbf{1} & 0 & -\mathbf{j}
\end{array}\right], \quad \phi_{3} \equiv\left[\begin{array}{c}
\mathbf{f}_{23} \\
T_{30} \\
f_{30}
\end{array}\right], \quad \mathbf{t}_{3} \equiv\left[\begin{array}{l}
0 \\
\dot{\mathbf{c}}
\end{array}\right], \quad \mathbf{w}_{3} \equiv\left[\begin{array}{c}
T_{3} \\
\mathbf{f}_{3}
\end{array}\right]
$$

Hence, using equation (7) for links 1 and 2, and equation (11) for link 3, the mathematical model is obtained:

$$
\mathbf{M} \dot{\mathbf{t}}=\mathbf{w}+\mathbf{B} \phi
$$

where the $9 \times 9$ matrix $\mathbf{M}$, the mechanism inertia dyad, and the $9 \times 8$ matrix $\mathbf{B}$, are given below:

$$
\begin{aligned}
\mathbf{M} & \equiv \operatorname{diag}\left(\mathbf{M}_{1}, \mathbf{M}_{2}, \mathbf{M}_{3}\right) \\
\mathbf{B} & \equiv\left[\begin{array}{ccccc}
\mathbf{r}_{1}^{T} \mathbf{E} & \mathbf{b}_{1}^{T} \mathbf{E} & \mathbf{0}^{T} & 0 & 0 \\
\mathbf{1} & -\mathbf{1} & \mathbf{O} & \mathbf{0} & \mathbf{0} \\
0^{T} & \mathbf{r}_{2}^{T} \mathbf{E} & \mathbf{b}_{2}^{T} \mathbf{E} & 0 & 0 \\
\mathbf{O} & \mathbf{1} & -\mathbf{1} & \mathbf{0} & \mathbf{0} \\
0^{T} & 0^{T} & \mathbf{r}_{3}^{T} \mathbf{E} & -1 & 0 \\
\mathbf{O} & \mathbf{O} & \mathbf{1} & \mathbf{0} & -\mathbf{j}
\end{array}\right]
\end{aligned}
$$




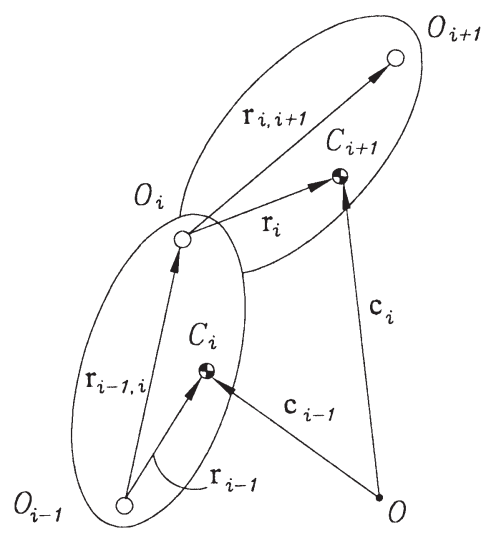

Fig. 4. Geometric relations between the (i-1)st and the ith links.

in which $\mathbf{0}$ is the two-dimensional zero vector, while $\mathbf{O}$ and $\mathbf{1}$ are the $2 \times 2$ zero matrix and the $2 \times 2$ identity matrix, respectively. Moreover the eight-dimensional vector $\phi$ is defined, in turn, as

$$
\phi \equiv\left[\mathbf{f}_{01}^{T}, \mathbf{f}_{12}^{T}, \mathbf{f}_{23}^{T}, T_{30}, f_{30}\right]^{T}
$$

Thus, $\phi$ is the array of internal or constraint wrench of the whole mechanism, which does not develop any power, its sole role being to keep all links together.

We can now write the kinematic constraint equations of the slider-crank. Referring to Fig. 4, the kinematic constraints between links 1 and 0;2 and 1; and 3 and 2 can be expressed as

$$
\begin{aligned}
& \omega_{i} \equiv \omega_{i-1}+\dot{\theta}_{i-1} \\
& \dot{\mathbf{c}}_{i} \equiv \dot{\mathbf{c}}_{i-1}+\omega_{i-\mathrm{I}} \mathbf{E} \mathbf{b}_{i-1}+\omega_{i} \mathbf{E r}_{i}
\end{aligned}
$$

where $\dot{\mathbf{c}}_{i-1}$ and $\dot{\mathbf{c}}_{i}$ are the velocities of the mass centres of the $(i-1)$ st and the $i$ th links, respectively, while $\dot{\theta}_{0}=0, \dot{\theta}_{1}=\dot{\psi}, \dot{\theta}_{2}=\dot{\theta}$ and $\dot{\theta}_{3}$ is the relative angular velocity of the slider with respect to the connecting rod, i.e., $\dot{\theta}_{3}=-\dot{\theta}$. Now, equation (18) can be written in the form

$$
\mathbf{A}_{i, i-1} \mathbf{t}_{i-1}+\mathbf{A}_{i, i} \mathbf{t}_{i}=0 \quad i=1,2,0
$$

In equation (19), the $2 \times 3$ matrices $\mathbf{A}_{i, i-1}$ and $\mathbf{A}_{i, i}$, for $i=1,2,0$, are given as

$$
\begin{aligned}
\mathbf{A}_{i, i-1} & \equiv\left[\begin{array}{ll}
-\mathbf{E b}_{i-1} & -\mathbf{1}
\end{array}\right] \\
\mathbf{A}_{i, i} & \equiv\left[\begin{array}{ll}
-\mathbf{E r}_{i} & \mathbf{1}
\end{array}\right]
\end{aligned}
$$


The constraints between the slider and the base, links 3 and 0 , can be written in turn as

$$
\omega_{3}=0 \text { and } \mathbf{j}^{T} \dot{\mathbf{c}}_{3}=0
$$

since link 3 undergoes a pure translation in the direction of vector $\mathbf{i}$. Combining equation (19) for $i=1,2,0$, with the foregoing constraints, equation (22), the kinematic constraints for the mechanism under study, become then a linear homogeneous system:

$$
\mathbf{A t}=\mathbf{0}
$$

where $\mathbf{t}=\left[\mathbf{t}_{1}^{T}, \mathbf{t}_{2}^{T}, \mathbf{t}_{3}^{T}\right]^{T}$ and the $8 \times 9$ matrix $\mathbf{A}$ is defined as

$$
\mathbf{A} \equiv\left[\begin{array}{cccccc}
-\mathbf{E r}_{1} & \mathbf{1} & \mathbf{0} & \mathbf{O} & \mathbf{0} & \mathbf{O} \\
-\mathbf{E} \mathbf{b}_{1} & -\mathbf{1} & -\mathbf{E r}_{2} & \mathbf{1} & \mathbf{0} & \mathbf{O} \\
\mathbf{0} & \mathbf{O} & -\mathbf{E} \mathbf{b}_{2} & -\mathbf{1} & -\mathbf{E} \mathbf{r}_{3} & \mathbf{1} \\
0 & \mathbf{0}^{T} & 0 & 0^{T} & -1 & \mathbf{0}^{T} \\
0 & \mathbf{0}^{T} & 0 & 0^{T} & 0 & -\mathbf{j}^{T}
\end{array}\right]
$$

It is apparent that $\mathbf{A}^{T}=\mathbf{B}$, which is not by chance. This is a result of the reciprocity between the feasible twists of the cnstrained mechanical system and the constraint wrenches exerted by the joints. In fact, upon rewriting equation (13) in terms of $\mathbf{A}$,

$$
\mathbf{M} \dot{\mathbf{t}}=\mathbf{w}+\mathbf{A}^{T} \phi
$$

it becomes apparent that the internal wrench, $\phi$, is nothing but the vector of Lagrange multipliers of classical dynamics. Furthermore, the twists of the individual links are given as

$$
\mathbf{t}_{1} \equiv \dot{\psi}\left[\begin{array}{c}
1 \\
\mathbf{E} \mathbf{r}_{1}
\end{array}\right], \quad \mathbf{t}_{2} \equiv\left[\begin{array}{c}
\dot{\theta} \\
\dot{\psi} \mathbf{E r}_{12}+\dot{\theta} \mathbf{E} \mathbf{r}_{2}
\end{array}\right], \quad \mathbf{t}_{3} \equiv\left[\begin{array}{c}
0 \\
\mathbf{i} \dot{s}
\end{array}\right]
$$

Now we derive the mathematical model of the single-degree-of-freedom system, i.e., we project all individual, uncoupled $\mathrm{N}-\mathrm{E}$ equations $(5,6)$ onto the input revolute axis, the result being one single second-order ordinary differential equation. To do this, we set up first the loop-closure equation of the mechanism, with reference to Fig. 1:

$$
\mathbf{r}_{12}+\mathbf{r}_{23}+\mathbf{r}_{30}+\mathbf{r}_{01}=\mathbf{0}
$$

Upon differentiation of both sides of equation (27) with respect to time, we obtain the loop-velocity equation, namely,

$$
\mathbf{E}\left(\dot{\psi} \mathbf{r}_{12}+\dot{\theta} \mathbf{r}_{23}\right)+\dot{\mathbf{r}}_{01}=\mathbf{0}
$$


Moreover, the derivatives of $\mathbf{r}_{01}$ and $\theta$ are readily calculated as

$$
\frac{d \mathbf{r}_{01}}{d t}=-\mathbf{i} \dot{s}(t)=-\mathbf{i} r_{s} \dot{\psi}, \quad \dot{\theta}=\theta^{\prime}(\psi) \dot{\psi}
$$

where $r_{s}$ is an influence coefficient [22]. Then, using equation (29), the foregoing equation takes the form

$$
\mathbf{E}\left(\mathbf{r}_{12}+r_{0} \mathbf{r}_{23}\right)-\mathbf{i} r_{s}=\mathbf{0}
$$

In the above equation we have deleted the common factor $\dot{\psi} \neq 0$, thereby obtaining a two-dimensional vector equation in two unknowns, $r_{\theta}$ and $r_{s}$, with $r_{\theta}$ being a second influence coefficient. While the two foregoing coefficients can be calculated graphically [22], we outline here an algebraic calculation. First and foremost, we notice the important properties of matrix $\mathbf{E}$ below. If $\mathbf{r}$ is an arbitrary two-dimensional vector, then

$$
\mathbf{r}^{T} \mathbf{E r}=0, \quad \mathbf{E i}=\mathbf{j}, \quad \mathbf{E} \mathbf{j}=-\mathbf{i}
$$

Next, upon multiplying both sides of equation (30) from the left by $\mathbf{j}^{T}$ and $\mathbf{r}_{23}^{T}$, successively, we obtain the expressions for the influence coefficients as

$$
r_{\theta}=-\frac{\mathbf{i}^{T} \mathbf{r}_{12}}{\mathbf{i}^{T} \mathbf{r}_{23}}, \quad r_{s}=\frac{\mathbf{r}_{23}^{T} \mathbf{E r}_{12}}{\mathbf{r}_{23}^{T} \mathbf{i}}
$$

Once the influence coefficients are determined, the angular velocity $\dot{\theta}$ and the velocity $\dot{s}$ can be calculated as

$$
\dot{\theta}=r_{\theta} \dot{\psi}, \quad \dot{s}=r_{s} \dot{\psi}
$$

Knowing $\dot{\theta}$ and $\dot{s}$, the nine-dimensional generalised twist can be expressed as a linear transformation of $\dot{\psi}$ as

$$
\mathbf{t}=\mathbf{u} \dot{\psi}
$$

where vector $\mathbf{u}$ is defined as

$$
\mathbf{u} \equiv\left[\begin{array}{lllll}
1, & -\mathbf{r}_{1}^{T} \mathbf{E}, & r_{\theta}, & -\left(\mathbf{r}_{12}+r_{\theta} \mathbf{r}_{2}\right)^{T} \mathbf{E}, \quad 0, \quad r_{s} \mathbf{i}^{T}
\end{array}\right]^{T}
$$

Moreover, by virtue of equation (30), vector $\mathbf{u}$ satisfies the relation

$$
\mathbf{A u}=\mathbf{0}
$$

with $\mathbf{0}$ indicating the eight-dimensional zero vector, and, hence, $\mathbf{u}$ lies in the nullspace of A. (The concept of nullspace is central to linear algebra, to which mechanical engineering students are exposed at McGill University in their third semester.) By virtue of the definition of $\mathbf{A}$ and the vector of non-working constraint wrenches, after multiplication of both sides of equation (13) by $\mathbf{u}^{T}$, the vector of non-working constraint wrenches is eliminated from the said equations, and hence, 


$$
\mathbf{u}^{T} \mathbf{M} \dot{\mathbf{t}}=\mathbf{u}^{T} \mathbf{w}
$$

Moreover, upon differentiating both sides of equation (34) with respect to time, we have

$$
\dot{\mathbf{t}}=\mathbf{u} \ddot{\psi}+\dot{\mathbf{u}} \dot{\psi}
$$

Further, substitution of equation (38) into equation (37) leads to the mathematical model of the mechanism, namely,

$$
I(\psi) \ddot{\psi}=C(\dot{\psi}, \psi) \dot{\psi}+\tau
$$

where

$I(\psi) \equiv \mathbf{u}^{T} \mathbf{M u}$ (generalized inertia);

$C(\psi, \dot{\psi}) \dot{\psi} \equiv-\mathbf{u}^{T} \mathbf{M} \dot{\mathbf{u}} \dot{\psi}$ (Coriolis and centrifugal force terms);

$\tau(\psi) \equiv \mathbf{u}^{T} \mathbf{w}$ (generalized external force arising from actuation, gravity and dissipation effects).

Furthermore, the generalized inertia of the mechanism is given by

$$
I(\psi)=I_{1}+m_{1}\left\|\mathbf{r}_{1}\right\|^{2}+r_{\theta}^{2} I_{2}+m_{2}\left\|\mathbf{r}_{12}+r_{\theta} \mathbf{r}_{2}\right\|^{2}+m_{3} r_{s}^{2}
$$

which can also be derived from the well known theorem of parallel axes of firstyear mechanics, as applied to a composite body, comprising all moving links. This interpretation of the generalised inertia of the mechanism should explain why this concept is configuration-dependent. Moreover, the coefficient, $C(\psi, \dot{\psi})$, of the Coriolis and centrifugal forces is given by

$$
C(\psi, \dot{\psi})=-\dot{\psi}\left\{r_{\theta} r_{\theta}^{\prime} I_{2}+m_{2}\left[r_{\theta}^{\prime}\left(\mathbf{E r}_{12}+r_{\theta} \mathbf{r}_{2}\right)^{T} \mathbf{r}_{2}+r_{\theta}\left(\mathbf{r}_{\theta}-1\right) \mathbf{r}_{12}^{T} \mathbf{E} \mathbf{r}_{2}\right]+m_{3} r_{s} r_{s}^{\prime}\right\}
$$

where $\|\cdot\|$ is the Euclidean norm of vector $(\cdot)$, while $r_{\theta}^{\prime}$ and $r_{s}^{\prime}$ are defined as

$$
r_{\theta}^{\prime} \equiv r_{\theta}^{\prime}(\psi) \equiv \frac{d r_{\theta}}{d \psi} \equiv \frac{\dot{r}_{\theta}}{\dot{\psi}}, \quad r_{s}^{\prime} \equiv r_{s}^{\prime}(\psi) \equiv \frac{d r_{s}}{d \psi} \equiv \frac{\dot{r}_{s}}{\dot{\psi}},
$$

Furthermore, the external generalized torque, $\tau$, is given by

$$
\tau(\psi) \equiv \tau^{A}+\tau^{G}+\tau^{D}
$$

where $\tau^{A}$ is the driving torque that is assumed to be applied at link 1 by the driving motor, while $\tau^{G}$ and $\tau^{D}$ account for the gravity and dissipative effects of the system, respectively. As to the latter, and in light of our assumption of ideal joints, only viscous effects are considered here.

\section{Dynamic balancing}

The dynamic balancing of the slider-crank mechanism is now undertaken by resorting to the approach introduced by Angeles and Sinatra [23]. With reference to Fig. 2 , the centre of the $i$ th joint and the mass centre of the $i$ th link are denoted by $O_{i}$ 
and $C_{i}$, for $i=0,1,2,3$, respectively. Note that only the mass centres of the moving bodies are relevant.

We now introduce the definitions below:

$$
m=\sum_{i=1}^{3} m_{i}, \quad m \mathbf{c}=\sum_{i=1}^{3} m_{i} \mathbf{c}_{i}
$$

i.e., $m$ is the total mass of the linkage, and $\mathbf{c}$ is the position vector of the mass centre $C$ of the linkage. Upon differentiation of both sides of equation (44) with respect to time, we obtain

$$
m \dot{\mathbf{c}} \equiv \sum_{i=1}^{3} m_{i} \dot{\mathbf{c}}_{i}
$$

One more differentiation with respect to time yields

$$
m \ddot{\mathbf{c}} \equiv \sum_{i=1}^{3} m_{i} \ddot{\mathbf{c}}_{i}
$$

The N-E equation (6) is now recalled, and written for all three moving links:

$$
\begin{gathered}
\mathbf{f}_{01}+\mathbf{f}_{1}-\mathbf{f}_{12}=m_{1} \ddot{\mathbf{c}}_{1} \\
\mathbf{f}_{12}+\mathbf{f}_{2}-\mathbf{f}_{23}=m_{2} \ddot{\mathbf{c}}_{2} \\
\left(\mathbf{f}_{23}+\mathbf{f}_{3}\right)^{T} \mathbf{i}=m_{3} \ddot{s} \\
\left(\mathbf{f}_{23}+\mathbf{f}_{3}\right)^{T} \mathbf{j}-f_{30}=0
\end{gathered}
$$

The sum of all foregoing equations leads to

$$
\mathbf{f}_{01}+\mathbf{f}_{1}+\mathbf{f}_{2}+\mathbf{f}_{3}-f_{30} \mathbf{j}=m_{1} \ddot{\mathbf{c}}_{1}+m_{2} \ddot{\mathbf{c}}_{2}+m_{3} \ddot{\mathbf{c}}_{3}
$$

Further, we introduce equation (46) into the above equation, and hence,

$$
\mathbf{f}_{01}-f_{30} \mathbf{j}=m \ddot{\mathbf{c}}-\left(\mathbf{f}_{1}+\mathbf{f}_{2}+\mathbf{f}_{3}\right)
$$

As in the case of the four-bar linkage, dynamic balancing is achieved if the mass centre of the linkage is stationary, i.e., if

$$
m \ddot{\mathbf{c}}=\mathbf{0}
$$

which implies

$$
m \dot{\mathbf{c}}=\text { constant }
$$

the above constant being necessarily equal to zero due the limited mobility of the linkage. Hence, the balancing condition can be stated as

$$
m_{1} \dot{\mathbf{c}}_{1}+m_{2} \dot{\mathbf{c}}_{2}+m_{3} \dot{\mathbf{c}}_{3}=\mathbf{0}
$$


Now, from equation (34), the velocity vectors of the mass centres of all links are given as

$$
\dot{\mathbf{c}}_{1}=\dot{\psi} \mathbf{E} \mathbf{r}_{1}, \quad \dot{\mathbf{c}}_{2}=\dot{\psi}\left(\mathbf{E r}_{12}+r_{\theta} \mathbf{r}_{2}\right), \quad \dot{\mathbf{c}}_{3}=\dot{\psi} r_{s} \mathbf{i}
$$

Thus, upon substituting equation (55) into equation (56), we obtain

$$
\dot{\psi}\left[\left(m_{1}+m_{2}\right) \mathbf{E} \mathbf{r}_{1}+m_{2} \mathbf{E}\left(r_{\theta} r_{2}-\mathbf{r}_{1}+\mathbf{r}_{12}\right)+m_{3} r_{s} \mathbf{i}\right]=\mathbf{0}
$$

and, since $\dot{\psi} \neq 0$,

$$
\left(m_{1}+m_{2}\right) \mathbf{E} \mathbf{r}_{1}+m_{2} \mathbf{E}\left(r_{\theta} \mathbf{r}_{2}-\mathbf{r}_{1}+\mathbf{r}_{12}\right)+m_{3} r_{s} \mathbf{i}=\mathbf{0}
$$

Now, if we let

$$
\mu_{i}=\frac{m_{i}}{m_{3}}, \quad i=1,2
$$

then, equation (58) can be recast as

$$
\mathbf{E}\left(\mu_{1} \mathbf{r}_{1}+\mu_{2} \mathbf{r}_{12}+\mu_{2} r_{\theta} \mathbf{r}_{2}\right)+r_{s} \mathbf{i}=\mathbf{0}
$$

Multiplication of both sides of equation (60) by $\mathbf{E}$ leads to

$$
r_{s} \mathbf{j}-\left(\mu_{1} \mathbf{r}_{1}+\mu_{2} \mathbf{r}_{12}+\mu_{2} r_{\theta} \mathbf{r}_{2}\right)=\mathbf{0}
$$

On the other hand, equation (61) can be simplified if we recall equation (30), which allows us to express $\mathbf{i} r_{s}$ as a linear transformation of $\mathbf{r}_{12}+r_{\theta} \mathbf{r}_{23}$, thereby obtaining

$$
\mu_{1} \mathbf{r}_{1}+\left(1+\mu_{2}\right) \mathbf{r}_{12}+\mu_{2} r_{\theta} \mathbf{r}_{2}+r_{\theta} \mathbf{r}_{23}=\mathbf{0}
$$

Now, for arbitrary links, we can always express the local position vector of the mass centre of the $i$ th link as a linear transformation of the corresponding vector $\mathbf{r}_{i, i+1}$, i.e.,

$$
\mathbf{r}_{1}=\mathbf{C r}_{12}, \quad \mathbf{r}_{2}=\mathbf{D} \mathbf{r}_{23}
$$

where $\mathbf{C}$ and $\mathbf{D}$ are $2 \times 2$ matrices representing a rotation plus a scaling, namely,

$$
\mathbf{C}=\rho_{1}\left(\cos \alpha_{1} \mathbf{1}+\sin \alpha_{1} \mathbf{E}\right), \quad \mathbf{D}=\rho_{2}\left(\cos \alpha_{2} \mathbf{1}+\sin \alpha_{2} \mathbf{E}\right)
$$

where $\alpha_{i}$ denotes the corresponding angle of rotation, while $\rho_{i}$ denotes the concomitant scaling factor, for $i=1,2$. It is noteworthy that the $2 \times 2$ matrix inside the parentheses in the above expressions represents a rotation in the plane through an angle $\alpha_{i}$, for $i=1,2$ [24]. Moreover, the scaling factors can be readily found to be

$$
\rho_{i}=\frac{\left\|\mathbf{r}_{i}\right\|}{\left\|\mathbf{r}_{i, i+1}\right\|}, \quad i=1,2,3 \quad \text { modulo } 3
$$

Substitution of the expressions for $\mathbf{r}_{1}$ and of $\mathbf{r}_{2}$ given in equation (63), using equation (64), we can rewrite equation (62) as 


$$
\begin{aligned}
& {\left[\left(1+\mu_{1} \rho_{1} \cos \alpha_{1}+\mu_{2}\right) \mathbf{1}+\mu_{1} \rho_{1} \sin \alpha_{1} \mathbf{E}\right] \mathbf{r}_{12}+\left[\left(1+\mu_{2} \rho_{2} \cos \alpha_{2}\right) \mathbf{1}\right.} \\
& \left.\quad+\mu_{2} \rho_{2} \sin \alpha_{2} \mathbf{E} r_{\theta} \mathbf{r}_{23}\right]=\mathbf{0}
\end{aligned}
$$

In order to proceed further, we need to impose the condition that the coefficients of $\mathbf{1}$ and of $\mathbf{E}$ inside the brackets in the above equation be equal, i.e.,

$$
\begin{aligned}
& \mu_{1} \rho_{1} \cos \alpha_{1}+\mu_{2}=\mu_{2} \rho_{2} \cos \alpha_{2} \\
& \mu_{1} \rho_{1} \sin \alpha_{1}=\mu_{2} \rho_{2} \sin \alpha_{2}
\end{aligned}
$$

Thus, equation (60) can be simplified as

$$
\left[\left(1+\mu_{1} \rho_{1} \cos \alpha_{1}+\mu_{2}\right) \mathbf{1}+\mu_{1} \rho_{1} \sin \alpha_{1} \mathbf{E}\right]\left(\mathbf{r}_{12}+r_{\theta} \mathbf{r}_{23}\right)=\mathbf{0}
$$

Moreover, note that $\mathbf{E}^{2}=\mathbf{- 1}$; additionally, using equation (62), the foregoing relation reduces to

$$
\left[\left(1+\mu_{1} \rho_{1} \cos \alpha_{1}+\mu_{2}\right) \mathbf{E}-\mu_{1} \rho_{1} \sin \alpha_{1} \mathbf{1}\right] \mathbf{i} r_{s}=0
$$

Now, by virtue of equation (29), $r_{s} \neq 0$, while $\mathbf{i} \neq \mathbf{0}$, for it is a basis unit vector. Hence, equation (70) leads to

$$
\begin{aligned}
& 1+\mu_{1} \rho_{1} \cos \alpha_{1}+\mu_{2}=0 \\
& \mu_{1} \rho_{1} \sin \alpha_{1}=0
\end{aligned}
$$

which, along with equations (67) and (68) are the dynamic balancing conditions sought. Note that these conditions involve six linkage parameters, namely, $\alpha_{1}, \alpha_{2}$, $\rho_{1}, \rho_{2}, \mu_{1}$, and $\mu_{2}$. In light of the definitions of these paramaters, however, the last four are constrained to be positive quantities, which, in an optimization problem, can become highly demanding for undergraduate students. Most likely, parameters $\mu_{1}$ and $\mu_{2}$ will be either dictated by the specific application at hand, or decided on by the designer based on experience. In order to avoid dealing with constrained variables, non-dimensional Cartesian coordinates can be introduced, namely,

$$
\xi_{i}=\rho_{i} \cos \alpha_{i}, \quad \eta_{i}=\rho_{i} \sin \alpha_{i}, \quad i=1,2
$$

which can attain unbounded real values. By virtue of equations (68) and (72), we have

$$
\eta_{1}=\eta_{2}=0
$$

while the remaining coordinates can be determined from equations (67) and (71), i.e.,

$$
\xi_{1}=-\frac{1+\mu_{2}}{\mu_{1}}, \quad \xi_{2}=\frac{1}{\mu_{2}}
$$


Therefore, if the mass ratios are given, then the non-dimensional Cartesian cordinates of the mass centres of the various links, after balancing, are given by equations (74) and (75).

\section{Conclusions}

The dynamics of the slider-crank mechanism was formulated using a novel, systematic approach. This approach allowed a straightforward derivation of the dynamic balancing conditions of the foregoing mechanism. These conditions are expressed as two equations in six linkage parameters. A few concepts of linear algebra were invoked, which are nowadays standard components of the mechanical engineering undergraduate core curriculum. Students should therefore be expected to handle these concepts with some familiarity. Finally, it is noteworthy that, by manipulating vectors and matrices as blocks - a frame-invariant approach - as opposed to manipulating their components, first, physical insight is gained; then, all relations derived are produced in a more economic and more concise form than if components were used.

\section{Acknowledgements}

The research work reported here was possible under MURST (finanziamento PRIN anno 2000, codice progetto MM09113357-005).

\section{References}

[1] A. G. Erdman and G. N. Sandor, Mechanism Design: Analysis and Synthesis, Vol. 1 (Prentice Hall, Englewood Cliffs, NS, 1994).

[2] J. F. Gardner, Simulations of Machines Using Matlab and Simulink (Brooks Cole Publishing, Pacific Grove, CA, 2001).

[3] J. Angeles and S. Lee, 'The formulation of dynamical equations of holonomic mechanical systems using a natural orthogonal complement', ASME Journal of Applied Mechanics, 55 (1988), 243-244.

[4] J. Angeles and S. Lee, 'The modelling of holonomic mechanical systems using a natural orthogonal complement', Transactions of the Canadian Society of Mechanical Engineers, 13(4) (1989), 81-89.

[5] R. S. Berkof and G. G. Lowen, 'A new method for completely force balancing simple linkages', Transactions of the ASME, Ser. B, 91(1) (1969), 21-26.

[6] I. S. Kochev, 'A new general method for full force balancing of planar linkages', Mechanism and Machine Theory, 23(6) (1988), 475-480.

[7] C. Bagci, 'Shaking force balancing of planar linkages with force transmission irregularities using balancing idler loops', Mechanism and Machine Theory, 14(4) (1979), 267-284.

[8] G. Feng, 'Complete shaking force and shaking moment balancing of four types of six-bar linkages', Mechanism and Machine Theory, 24(4) (1989), 275-287.

[9] T. Thümmel, Dynamic Balancing of Linkages by Active Control with Redundant Drives, Technical Report (Department B of Mechanics, Technical University of Munich, Germany, 1994).

[10] J. Angeles, M. A. Nahon and T. Thümmel, 'Active control for the complete dynamic balancing of linkages', 22nd Biennal ASME Mechanisms Conference (Scottsdale, Arizona, 1992), vol. 47, Flexible Mechanisms, Dynamics and Analysis, pp. 305-310.

[11] M. R. Smith, 'Optimal balancing of planar multibar linkages', Proceedings of the 5th World Congress on the Theory of Machines and Mechanism (Newcastle-upon-Tyne, 1975), pp. 142-149. 
[12] F. Gao, 'Complete shaking force and shaking moment balancing of 17 types of eight-bar linkages', Mechanism and Machine Theory, 26(2) (1991), 179-206.

[13] M. Jean and C. M. Gosselin, 'Static balancing of planar parallel manipulators', Proceedings of the IEEE International Conference on Robotics and Automation (Minneapolis, Minnesota, 1996), pp. 3732-3737.

[14] C. Bagci, 'Complete balancing of space mechanisms - shaking force balancing', ASME Journal of Mechanisms, Transmissions, and Automation in Design, 105(12) (1983), 609-616.

[15] G. J. Walsh, D. A. Streit and B. J. Gilmore, 'Spatial spring equilibrator theory', Mechanism and Machine Theory, 26(2) (1991), 155-170.

[16] N. Ulrich and V. Kumar, 'Passive mechanical gravity compensation for robot manipulators', Proceedings of the IEEE International Conference on Robotics and Automation (Sacramento, CA, 1991), pp. 1536-1541.

[17] D. A. Streit and B. J. Gilmore, 'Perfect spring equilibrators for rotatable bodies', ASME Journal of Mechanisms, Transmissions and Automation in Design, 111(4) (1989), 451-458.

[18] C. M. Gosselin and J. Wang, 'On the design of gravity-compensated six-degree-of-freedom parallel mechanisms', Proceedings of the IEEE International Conference on Robotics and Automation (Leuven, Belgium, 1998), pp. 2287-2294.

[19] J. Wang and C. M. Gosselin, 'Static balancing of spatial three-degree-of-freedom parallel mechanisms', Mechanism and Machine Theory, 34 (1999), 437-452.

[20] J. Wang and C. M. Gosselin, 'Static balancing of spatial four-degree-of-freedom parallel mechanisms', Mechanism and Machine Theory, 35 (2000), 563-592.

[21] C. Bagci, 'Shaking force and shaking moment balancing of plane slider-crank mechanism', Proceedings of the 4th OSU Applied Mechanism Conference, (1975), pp. 1-25.

[22] J. Angeles and González-Palacios, 'The loop-polygon method of analysis of planar linkages', Proceedings of the sixth IFToMM International Symposium on Linkages and Computer Aided Design Methods - Theory and Practice (Bucharest, 1993), pp. 11-19.

[23] R. Sinatra and J. Angeles, 'A new tool in the teaching of planar mechanism and machine theory', Proceedings of the Ninth IFToMM International Conference (Milan, Italy, 1995), Vol. 4, pp. 3181-3186.

[24] O. Bottema and B. Roth, Theoretical Kinematics (Dover Publications, New York, 1979). 
Copyright $\odot 2003$ EBSCO Publishing 